\title{
POLYHEDRA AND OPTIMIZATION RELATED TO A WEAK ABSOLUTE MAJORIZATION ORDERING
}

\author{
Ping Zhan* \\ Edogawa University
}

(Received May 28, 2003; Revised September 30, 2004)

\begin{abstract}
A vector $x \in \mathbf{R}^{n}$ is weakly $k$-majorized by a vector $q \in \mathbf{R}^{k}$ if the sum of $r$ largest components of $x$ is less than or equal to the sum of $r$ largest components of $q$ for $r=1,2, \cdots, k$ and $k \leq n$. In this paper we extend the components of $x$ to their absolute values in the above description and generalize some results in [2] and [3] by G. Dahl and F. Margot.
\end{abstract}

Keywords: Majorization, bisubmodular functions, polyhedra

\section{Introduction}

For $p, q \in \mathbf{R}^{n}$ we say that $p$ is weakly sub-majorized by $q$ if $\sum_{j=1}^{r} p_{[j]} \leq \sum_{j=1}^{r} q_{[j]}$ for $r=1,2, \cdots, n$. Here $p_{[j]}$ denotes the $j$ th largest component of $p$. If $\sum_{j=1}^{n} p_{j}=\sum_{j=1}^{n} q_{j}$ also holds, $p$ is majorized by $q$ and we write $p \prec q$. Furthermore, for integers $k, n$ with $k \leq n$ and $x \in \mathbf{R}^{n}, q \in \mathbf{R}^{k}$, we say that $x$ is weakly $k$-majorized by $q$ and write $x \prec_{k} q$ if

$$
\sum_{j=1}^{r} x_{[j]} \leq \sum_{j=1}^{r} q_{[j]} \text { for } r=1,2, \cdots, k .
$$

Majorization is a concept appearing in several branches of mathematics and applied mathematics as indicated in [2]. Here, we extend the components of $x$ in (1.1) to their absolute values as follows:

$$
\sum_{j=1}^{r}\left|x_{[j]}\right| \leq \sum_{j=1}^{r} q_{[j]} \text { for } r=1,2, \cdots, k .
$$

We say that $x$ is weakly absolutely $k$-majorized by $q$ and write $x_{\mathrm{abs}} \prec_{k} q$. In the following we investigate properties induced by (1.2) and generalize some results in [2] and [3].

Hereafter, we assume that majorant $q \in \mathbf{R}^{k}$ satisfies

$$
q_{1} \geq q_{2} \geq \cdots \geq q_{k} \geq 0
$$

\section{Polyhedra Induced by Absolute Majorization}

Let

$$
P(q ; k):=\left\{x \in \mathbf{R}^{n} \mid x \prec_{k} q\right\},
$$

and denote $\mathbf{N}_{t}:=\{1,2, \cdots, t\}$. Define $x(X):=\sum_{j \in X} x_{j}$. Then we have [2]

$$
P(q ; k)=\left\{x \in \mathbf{R}^{n} \mid x(X) \leq q\left(\mathbf{N}_{r}\right) \text { for all } X \subseteq \mathbf{N}_{n} \text { with } r=|X| \leq k\right\},
$$


so $P(q ; k)$ is a polyhedron.

In the case of absolute majorization, we first denote by $3^{\mathbf{N}_{n}}$ the set of all ordered pairs of disjoint subsets of $\mathbf{N}_{n}$, i.e., $3^{\mathbf{N}_{n}}:=\left\{(X, Y) \mid X, Y \subseteq N_{n}, X \cap Y=\emptyset\right\}$. Similarly, let

$$
P_{S}(q ; k):=\left\{x \in \mathbf{R}^{n} \mid x_{\mathrm{abs}} \prec_{k} q\right\}
$$

Then we have

$$
\begin{array}{r}
P_{S}(q ; k)=\left\{x \in \mathbf{R}^{n} \mid x(X)-x(Y) \leq q\left(\mathbf{N}_{r}\right)\right. \text { for all } \\
\left.(X, Y) \in 3^{\mathbf{N}_{n}} \text { with } r=|X \cup Y| \leq k\right\} .
\end{array}
$$

Hence $P_{S}(q ; k)$ is also a polyhedron, or more precisely, a polytope. Note here that the absolute-value notation disappears, and instead we have a signed linear form description.

Define the set function $f: 3^{\mathbf{N}_{n}} \rightarrow \mathbf{R}$ by

$$
f(X, Y)=\sum_{i=1}^{r} q_{i} \quad \text { for all }(X, Y) \in 3^{\mathbf{N}_{n}} \text { where } r=|X \cup Y|
$$

We call a function $f: 3^{\mathbf{N}_{n}} \rightarrow \mathbf{R}$ bisubmodular if $f$ satisfies

$f\left(X_{1}, Y_{1}\right)+f\left(X_{2}, Y_{2}\right) \geq f\left(X_{1} \cap X_{2}, Y_{1} \cap Y_{2}\right)+f\left(\left(X_{1} \cup X_{2}\right)-\left(Y_{1} \cup Y_{2}\right),\left(Y_{1} \cup Y_{2}\right)-\left(X_{1} \cup X_{2}\right)\right)$.

for any $\left(X_{1}, Y_{1}\right),\left(X_{2}, Y_{2}\right) \in 3^{\mathbf{N}_{n}}$.

Proposition 2.1 The function $f$ defined by (2.5) is a bisubmodular function.

(Proof) For any $\left(X_{1}, Y_{1}\right),\left(X_{2}, Y_{2}\right) \in 3^{\mathbf{N}_{n}}$, we show that the function satisfies bisubmodular inequality (2.6).

Rewrite the inequality (2.6) as

$f\left(X_{1}, Y_{1}\right)-f\left(X_{1} \cap X_{2}, Y_{1} \cap Y_{2}\right) \geq f\left(\left(X_{1} \cup X_{2}\right)-\left(Y_{1} \cup Y_{2}\right),\left(Y_{1} \cup Y_{2}\right)-\left(X_{1} \cup X_{2}\right)\right)-f\left(X_{2}, Y_{2}\right)$.

Let $s=\left|X_{1} \cap X_{2}\right|+\left|Y_{1} \cap Y_{2}\right|, t=\left|X_{2}\right|+\left|Y_{2}\right|$, and $l_{1}=\left|X_{1} \cup Y_{1}-X_{2} \cup Y_{2}\right|, l_{2}=\left|X_{1} \cap Y_{2}\right|+\left|X_{2} \cap Y_{1}\right|$. By the definition of function $f(X, Y)$ in (2.5), the left-hand side of $(2.7)$ is

$$
\sum_{i=s+1}^{s+l_{1}+l_{2}} q_{i}
$$

and the right-hand side of $(2.7)$ is

$$
\begin{cases}\sum_{i=t+1}^{t+l_{1}-l_{2}} q_{i} & \left(\text { if } l_{1} \geq l_{2}\right) \\ \sum_{i=t+l_{2}-l_{1}+1}^{t}-q_{i} & \left(\text { if } l_{1}<l_{2}\right)\end{cases}
$$

Since $t \geq s$, it follows from the assumption (1.3) that the left-hand side of (2.7) is, indeed, greater than or equal to the right-hand side of (2.7) (see Figure 1). Here note that $\left|L_{1}^{\prime} \cup L_{1}^{\prime \prime}\right|=$ $l_{1}$ and $\left|L_{2}^{\prime} \cup L_{2}^{\prime \prime}\right|=l_{2}$. 


$$
\begin{aligned}
& \frac{|\longleftarrow| X_{1} \cap X_{2}|+| Y_{1} \cap Y_{2}\left|+l_{1}+l_{2} \longrightarrow\right|}{|\longleftarrow| X_{1}|+| Y_{1}|\longrightarrow|} \\
& \frac{|\leftarrow| X_{1} \cup X_{2}-Y_{1} \cup Y_{2}|+| Y_{1} \cup Y_{2}-X_{1} \cup X_{2}|\rightarrow|}{|\longleftarrow| X_{2}|+| Y_{2}\left|+l_{1}-l_{2} \longrightarrow\right|}
\end{aligned}
$$

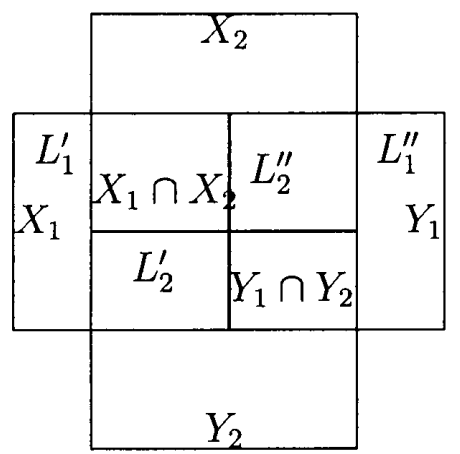

Figure 1: Lengths of sets and Venn diagram of Proposition 2.1

From the above proposition, we have a diameter description of $P_{S}(q ; n)$.

Let $P$ be a convex polytope. The diameter of $P$, denoted by $\delta(P)$, is the smallest number $\delta(P)$ such that any two vertices in $P$ can be connected by a path with at most $\delta(P)$ edges. Let $\phi$ be a linear form defined on $P$. Suppose that any $v$ can be connected to $v^{*}$ by a nonincreasing (nondecreasing) path induced by $\phi$ with at most $\delta(P, \phi)$ edges, where $v^{*}$ minimizes (maximizes) $\phi v$. The monotonic diameter of $P$ is defined as

$$
\delta^{*}(P)=\max \{\delta(P, \phi) \mid \phi \text { is a linear form on } P\} .
$$

Now we have the following result ([5], [1]).

Proposition 2.2 If all the components of $q$ take different values, the diameter and the monotonic diameter of $P_{S}(q, n)$ are $n^{2}$.

(Outline of the proof): From the proof of 2.1, we can easily see that different components of $q$ guarantee strict inequalities of bisubmodular function. Therefore, each vertex of $P_{S}(q ; n)$ can be characterized by a signed chain, a list of signed elements, or a special signed Hasse diagram [1]. Then we traverse to adjacent vertices along the edges of $P_{S}(q ; n)$ by two types of operations.

(1) Exchange the pair of two adjacent elements of a chain.

(2) Change the sign of an element at one fixed terminal of a chain.

Hence, what we have to do is counting the number of operations of types (1) and (2). Without loss of generality, we suppose that a maximally distant pair of vertices are characterized by following two chains.

$$
\begin{array}{r}
\left(-x_{1},-x_{2}, \cdots,-x_{n}\right) \\
\left(x_{1}, x_{2}, \cdots, x_{n}\right) .
\end{array}
$$

Hence, the total number of operations to obtain from one chain to another is

$$
1+(2 \times 2-1)+\cdots+(2 i-1)+\cdots+(2 n-1)=n^{2} .
$$

The monotonic property can be obtained from the definition of the bisubmodularity inequality.

\section{Optimization Problems and Integrality}

Let $c \in \mathbf{R}^{n}$ and consider the following optimization problem,

$$
\max \left\{c^{T} x \mid x_{\mathrm{abs}} \prec_{k} q\right\} \text {. }
$$


We know from (2.4) that the above problem is equivalent to

$$
\max \left\{c^{T} x \mid x \in P_{S}(q ; k)\right\},
$$

a linear programming (LP). And it is clear that when $k=n$, i.e., in the bisubmodular case, the above LP can be solved by greedy algorithm [4].

In the following, we generalize some results in [3] and [2] for $k<n$.

First, for $g \in \mathbf{R}^{k}$, we define tail average of $g$ by $\bar{g}_{s: k}:=1 /(k-s+1) \sum_{i=s}^{k}\left|g_{i}\right|$.

Suppose that $c \in \mathbf{R}^{k}$ satisfies $\left|c_{1}\right| \geq\left|c_{2}\right| \geq \cdots \geq\left|c_{k-1}\right|$ (note that $c_{k}$ may be arbitrary). Then there is an $m \in\{1,2, \cdots, k\}$ such that [2]

$$
\bar{c}_{1: k} \geq \cdots \geq \bar{c}_{m: k} \leq \bar{c}_{m+1: k} \leq \cdots \leq \bar{c}_{k: k}=\left|c_{k}\right|
$$

For $0 \leq s \leq k-1$ and $c \in \mathbf{R}^{n}$ with $\left|c_{1}\right| \geq\left|c_{2}\right| \geq \cdots \geq\left|c_{n}\right|$, we define signed sth q-average $w^{s} \in \mathbf{R}^{n}$ by

$$
w^{s}:=\left((-1)^{i_{1}} q_{1}, \cdots,(-1)^{i_{s}} q_{s},(-1)^{i_{s+1}} \bar{q}_{s+1: k}, \cdots,(-1)^{i_{n}} \bar{q}_{s+1, k}\right),
$$

where $i_{l}=0$ or 1 for $l=1,2, \cdots, n$, and define $w_{c}^{s} \in \mathbf{R}^{n}$, signed sth $q$-average related to $c$ by

$$
w_{c}^{s}:=\left(\operatorname{sign}\left(c_{1}\right) q_{1}, \cdots, \operatorname{sign}\left(c_{s}\right) q_{s}, \operatorname{sign}\left(c_{s+1}\right) \bar{q}_{s+1: k}, \cdots, \operatorname{sign}\left(c_{n}\right) \bar{q}_{s+1, k}\right) .
$$

Theorem 3.1 The optimal solution of Problem (3.2) can be obtained as a permutation of $w_{c}^{s}$, where $s=m-1$.

(Proof) To prove the optimality, we only consider the case when for $i=1,2, \cdots, n,(1)$ $x_{i} \geq 0$ if $c_{i} \geq 0$ and (2) $x_{i}<0$ if $c_{i}<0$. Otherwise, reversing the signs of components of $x$ would increase the value of $c^{\top} x$ without violating the constraints $x_{a b s} \prec_{k} q$. Our proof is only based on the form of $c^{T} x$, we may suppose $c_{i} \geq 0$ and $x_{i} \geq 0$ for $i=1,2, \cdots, n$. Otherwise, let $c_{i} x_{i}=\left(-c_{i}\right)\left(-x_{i}\right)$ and omit the signs before them for convenience.

For the second part of the proof, see appendix.

Conversely, by the same arguments as the proof of Theorem 3.1 and by an appropriate choice of $c$ (see Theorem 5 of [3]), we can prove that each $w^{s}(s=0,1, \cdots, k-1)$ is a unique optimal solution of $(3.2)$ on $P_{S}(q ; k)$, and therefore a vertex of $P_{S}(q ; k)$. Combining it with Theorem 3.1, we have the following result.

Theorem 3.2 The set of vertices of $P_{S}(q ; k)$ is precisely the set of vectors that can be obtained by permutations of $w^{s}, s=0,1, \cdots, k-1$.

By Theorem 3.1, the simple (greedy) algorithm for solving problem (3.1) is similar to that described in [2] and its time complexity is $O\left(n^{2}\right)$. The difference is that we compute $s$ by taking absolute values of components of $c$ and we take minus components of $w^{s}$ when the corresponding components of $c$ are less than zero.

Now we consider the following integer programming problem:

$$
\max \left\{c^{T} x \mid x_{\mathrm{abs}} \prec_{k} q, x \text { is integral }\right\} .
$$

We represent the integer hull of $P_{S}(q ; k)$ by

$$
Q_{S}(q ; k):=\operatorname{conv}\left\{x \in \mathbf{R}^{n} \mid x_{\text {abs }} \prec_{k} q, x \text { is integral }\right\} .
$$

We assume that $q$ is an integer vector, otherwise round down each component of $q$ without changing $Q_{S}(q ; k)$. Note here that $Q_{S}(q ; k)$ is full-dimensional if $q \neq 0$ (together with the assumption that $q$ is integral and non-negative). 
In [3] and [2], a complete description of vertices and facets of polyhedra $Q(q ; k)=$ $\operatorname{conv}\left\{x \in R^{n} \mid x \prec_{k} q, x\right.$ is integral $\}$ are provided. By the symmetry, these results can be generalized to polytope $Q_{S}(q ; k)$ without much modifications. We summarize two main results here.

Let $\alpha \in\left[q_{k}, \bar{q}_{1, k}\right]$ and define $s(\alpha)=\max \left\{0 \leq s<k \mid \bar{q}_{s+1, k} \geq \alpha\right\}$ and $\Delta(\alpha)=\sum_{i=s+1}^{k} q_{i}$ $(k-s(\alpha)-1) \alpha$. We define the vector $x(\alpha) \in \mathbf{R}^{n}$ by

$$
x(\alpha):=\left((-1)^{i_{1}} q_{1}, \cdots,(-1)^{i_{s}} q_{s(\alpha)},(-1)^{i_{s+1}} \Delta(\alpha),(-1)^{i_{s+2}} \alpha, \cdots,(-1)^{i_{n}} \alpha\right)
$$

for $i_{l}=0$ or 1 and $l=1,2, \cdots, n$, where $\alpha \leq \bar{q}_{1, k}$ is a round down or round up of $\bar{q}_{s+1, k}$ and $s=0,1, \cdots, k-1$. And we say $x(\alpha)$ a signed rounded $q$-average.

Theorem 3.3 The set of vertices of $Q_{S}(q ; k)$ is precisely the set of vectors that can be obtained by permutations of signed rounded q-averages.

Let $s$ and $t$ be integers with $0 \leq s<k<t \leq n$, and put $\delta^{s}:=\sum_{i=s+1}^{k} q_{i}-(k-s)\left\lfloor\bar{q}_{s+1: k}\right\rfloor=$ $\sum_{i=s+1}^{k} q_{i} \bmod (k-s)$. Then, define $\alpha_{0}^{s, t}:=(t-k) /\left(k-s-\delta^{s}\right) \sum_{i=1}^{s} q_{i}+\sum_{i=1}^{k} q_{i}+(t-$ $k)\left\lfloor\bar{q}_{s+1: k}\right\rfloor$. We call

$$
\left(t-s-\delta^{s}\right) /\left(k-s-\delta^{s}\right)\left(x\left(X_{1}\right)-x\left(Y_{1}\right)\right)+x\left(X_{2}\right)-x\left(Y_{2}\right) \leq \alpha_{0}^{s, t}
$$

a signed q-average inequality if $\bar{q}_{s+1: k}$ is fractional, where $X_{1}, Y_{1}, X_{2}, Y_{2}$ are pairwise disjoint, $\left|X_{1} \cup Y_{1}\right|=s$ and $\left|X_{2} \cup Y_{2}\right|=t-s$.

We call

$$
x(X)-x(Y) \leq \sum_{i=1}^{r} q_{i} \quad \text { for } \quad(X, Y) \in 3^{\mathbf{N}_{n}},|X \cup Y|=r
$$

a signed set size inequality if $r=1$ or $q_{1}>q_{r}$.

Theorem 3.4 A complete and non-redundant facet description of $Q_{S}(q ; k)$ is given by signed set size inequalities and signed q-average inequalities.

\section{Appendix}

For the second part of the proof of Theorem 3.1 we first assume $c_{1} \geq c_{2} \geq \cdots \geq c_{n}$. Let $x \geq 0$ be a feasible solution. Without loss of generality, we may assume that $x_{1} \geq \cdots \geq$ $x_{k}=\cdots=x_{n}$. Let $d_{i}=c_{i}$ for $i=1,2, \cdots, k-1$, and $d_{k}=\sum_{j=k}^{n} c_{j}$. Let $x^{*}=w_{c}^{s}$, as indicated in the theorem.

$$
\begin{aligned}
& \sum_{i=1}^{n} c_{i}\left(x_{i}^{*}-x_{i}\right)=\sum_{i=1}^{k} d_{i}\left(x_{i}^{*}-x_{i}\right)=\sum_{i=1}^{s} d_{i}\left(q_{i}-x_{i}\right)+\bar{q}_{s+1: k} \sum_{i=s+1}^{k} d_{i}-\sum_{i=s+1}^{k} d_{i} x_{i} \\
& =\sum_{i=1}^{s} d_{i}\left(q_{i}-x_{i}\right)+(k-s) \bar{d}_{s+1: k} \bar{q}_{s+1: k}-\sum_{i=s+1}^{k} d_{i} x_{i},
\end{aligned}
$$


where,

$$
\begin{aligned}
& -\sum_{i=s+1}^{k} d_{i} x_{i}=-\sum_{i=s+1}^{k-1}\left(\sum_{j=i}^{k} d_{j}-\sum_{j=i+1}^{k} d_{j}\right) x_{i}-d_{k} x_{k} \\
& =-\sum_{i=s+1}^{k-1}\left((k-i+1) \bar{d}_{i: k}-(k-i) \bar{d}_{i+1: k}\right) x_{i}-d_{k} x_{k} \\
& =-\left(\sum_{i=s}^{k-2}(k-i) \vec{d}_{i+1: k} x_{i+1}-\sum_{i=s+1}^{k-1}(k-i) \bar{d}_{i+1: k} x_{i}\right)-d_{k} x_{k} \\
& =\sum_{i=s+1}^{k-2}(k-i) \bar{d}_{i+1: k}\left(x_{i}-x_{i+1}\right)-(k-s) \bar{d}_{s+1: k} x_{s+1}+\bar{d}_{k: k} x_{k-1}-d_{k} x_{k} .
\end{aligned}
$$

Note that $x_{i}-x_{i+1} \geq 0$. From the definition of $s$ we obtain

$$
\begin{aligned}
& \sum_{i=s+1}^{k-2}(k-i) \bar{d}_{i+1: k}\left(x_{i}-x_{i+1}\right) \\
& \geq \bar{d}_{s+1: k} \sum_{i=s+1}^{k-2}(k-i)\left(x_{i}-x_{i+1}\right) \\
& =\bar{d}_{s+1: k} \sum_{i=s+1}^{k-2}(k-i) x_{i}-\bar{d}_{s+1: k} \sum_{i=s+2}^{k-1}(k-i+1) x_{i} \\
& =-\bar{d}_{s+1: k} \sum_{i=s+2}^{k-2} x_{i}+(k-s-1) \bar{d}_{s+1: k} x_{s+1}-2 \bar{d}_{s+1: k} x_{k-1} \\
& =-\bar{d}_{s+1: k} \sum_{i=s+1}^{k-1} x_{i}+(k-s) \bar{d}_{s+1: k} x_{s+1}-\bar{d}_{s+1: k} x_{k-1} .
\end{aligned}
$$

Now, the last whole row of equation (4.2) (after deleting $\left.(k-s) \bar{d}_{s+1: k} x_{s+1}-(k-s) \bar{d}_{s+1: k} x_{s+1}\right)$ ) is greater than

$$
\begin{aligned}
& -\bar{d}_{s+1: k} \sum_{i=s+1}^{k-1} x_{i}-\bar{d}_{s+1: k} x_{k-1}+\bar{d}_{k: k} x_{k-1}-d_{k} x_{k} \\
& =-\bar{d}_{s+1: k} \sum_{i=s+1}^{k} x_{i}+\bar{d}_{s+1: k} x_{k}-\bar{d}_{s+1: k} x_{k-1}+\bar{d}_{k: k} x_{k-1}-d_{k} x_{k} \\
& =-\bar{d}_{s+1: k} \sum_{i=s+1}^{k} x_{i}+\left(d_{k}-\bar{d}_{s+1: k}\right)\left(x_{k-1}-x_{k}\right) \\
& \geq-\bar{d}_{s+1: k} \sum_{i=s+1}^{k} x_{i} .
\end{aligned}
$$

Finally, since $q_{i}-x_{i} \geq 0$ for all $i=1,2, \cdots, s$, we have

$$
\sum_{i=1}^{s} d_{i}\left(q_{i}-x_{i}\right) \geq \bar{d}_{s+1: k} \sum_{i=1}^{s}\left(q_{i}-x_{i}\right) \text {. }
$$


Summarizing the above equations and inequalities, we have

$$
\sum_{i=1}^{k} d_{i}\left(x_{i}^{*}-x_{i}\right) \geq \bar{d}_{s+1: k}\left(\sum_{i=1}^{k} q_{i}-\sum_{i=1}^{k} x_{i}\right) \geq 0 .
$$

Note that this is a direct proof of Theorem 2 in [2].

\section{Acknowledgement}

The author would like to thank Prof. Satoru Fujishige, Dr. Takeshi Naitoh and anonymous referees for their valuable comments.

\section{References}

[1] K. Ando and S. Fujishige: On structures of bisubmodular Polyhedra. Mathematical Programming, 74 (1996), 293-317.

[2] G. Dahl: Polyhedra and optimization in connection with a weak majorization ordering. In E. Balas, J. Clausen (eds.): Integer Programming and Combinatorial Optimization (Springer-Verlag, Berlin, New York, 1995), 426-437.

[3] G. Dahl and F. Margot: Weak k-majorization and polyhedra. Mathematical Programming, 81 (1998), 37-53.

[4] S. Fujishige: Submodular Functions and Optimization (North-Holland, Amsterdam, 1991).

[5] P. Zhan and K. Naitoh: A polynomial-time algorithm for enumerating all vertices of bisubmodular polyhedra. Submitted.

Ping Zhan

Edogawa University

474 Komaki, Nagareyama-shi, Chiba, 270-0198, Japan

E-mail: zhan@edogawa-u.ac.jp 